\title{
5. DISCUSSION FOLLOWING THE REPORTS \\ BY WEAVER AND FIELD
}

(Tuesday September 9, 1969)

Chairman: B. F. BuRKE

Editor's remark: This long, very intensive, and partially very confused discussion, has been rearranged in six sections: (1) Direct and Indirect Evidence of X-rays and Low-Energy Cosmic Rays; (2) The Boundary Layer between the Stable Gas Phases; (3) Theoretical Aspects of Interstellar Gas Dynamics and the Formation of Clouds; (4) Observational Aspects of Interstellar Gas Dynamics and the Formation of Clouds; (5) Observations of the Rarefied, Neutral Intercloud Medium and of the Interstellar Electron Density; (6) The Dynamical Theory of HII Regions. Section 2 has been transferred from the Discussion on Monday, September 8 (Chapter 2). To Section 6 have been added remarks made during various discussions. A couple of remarks have been transferred to other Discussions. Part of the Discussion (in Section 5) was very confused; an attempt has been made to condense and to make as much sense as possible out of what was said. For the convenience of the reader I recapitulate a few concepts, the (mis)-use of which lead partially to the confusion:

(i) The hydrogen surface density or column density $N_{\mathrm{H}}=\int n_{\mathrm{H}} \mathrm{d} l\left(N_{\mathrm{H}}\right.$ is sometimes called the hydrogen measure HM).

(ii) The dispersion measure $\mathrm{DM}=\int n_{e} \mathrm{~d} l$ (DM is often called the electron surface density $N_{e}$ ).

(iii) The rotation measure $\mathrm{RM}=c_{1} \int n_{e} B_{\|} \mathrm{d} l$.

(iv) The emission measure $\mathrm{EM}=\int n_{e}^{2} \mathrm{~d} l$.

In these definitions, $n_{\mathrm{H}}$ represents the hydrogen density, $n_{e}$ the electron density, $B_{\|}$ the component of the magnetic field strength along the line of sight and $l$ the distance along the line of sight. Conventionally $n_{\mathrm{H}}$ and $n_{e}$ are expressed in $\mathrm{cm}^{-3}, B$ in $\mu \mathrm{G}$ and $l$ in pc. In addition there are two combinations of these quantities (and of the electron temperature $T$ ) involved in the Discussion:

(v) The free-free absorption coefficient $k(v)=c_{2}(v) n_{e}^{2} T^{-3 / 2}$ ( $v$ is the frequency).

(vi) The free-free emissivity $\varepsilon(v)=c_{3}(v) n_{e}^{2} T^{-1 / 2}$ (only at radio wavelengths).

$c_{2}$ and $c_{3}$ depend also on $T$, but only rather weakly.

\section{Direct and Indirect Evidence of X-rays and Low-Energy Cosmic Rays}

Sunyaev: I want to discuss an observational upper limit on the number of low-energy cosmic rays. Cosmic-ray protons may become neutral atoms without loss of kinetic energy through charge-exchange reactions. Some of these fast atoms will be excited, either because the charge-exchange process leaves the atom in an excited state, or because the atom collides inelastically with thermal neutral atoms. The excited, fast 
atoms emit Doppler-shifted Ly- $\alpha$ quanta, for which the interstellar optical depth is low. Observation of such a Doppler-shifted flux then yields an upper limit on the cosmic-ray flux. Unfortunately the cross-sections get very small for cosmic-ray energies in excess of $100 \mathrm{keV}$. For particles in the energy range 25 to $100 \mathrm{keV}, \mathrm{Kurt}$ and I found an upper limit $W(E<100 \mathrm{keV})<5 \times 10^{-3} \mathrm{eV} \mathrm{cm}^{-3}$ from measurements by the 'Venus' space probe. To obtain a limit on the density of cosmic rays around $1 \mathrm{MeV}$ we have to interpolate between low and high energies. Suppose that all cosmic-ray protons are injected with energies $E>E_{0}(\gg 100 \mathrm{keV})$. The spectrum for $E<E_{0}$ is then determined by ionization losses and can be calculated. From $W(E<100 \mathrm{keV})<$ $5 \times 10^{-3} \mathrm{eV} \mathrm{cm}^{-3}$, it follows that $W\left(E<E_{0}\right)=(1$ to 3$) E_{0}^{3 / 2} \mathrm{eV} \mathrm{cm}^{-3}$, where $E_{0}$ is in $\mathrm{MeV}$.

Field: If the cosmic-ray heating is due to $2 \mathrm{MeV}$ particles, the required energy density is only $0.03 \mathrm{eV} \mathrm{cm}^{-3}, 30$ to 100 times less than Sunyaev's upper limit.

Sunyaev: The upper limit, which I mentioned, can be brought down considerably by narrowing the filter of the photometer. $100 \mathrm{keV}$ corresponds to a $17 \AA$ wide band, ten times smaller than the band in the existing experiments.

Silk: Let me make a few more comments on the low-energy cosmic-ray density. Direct observations of these particles have been made, but the numbers found have to be demodulated to correct for the effects of the solar wind. I compare two different demodulations: that by Gloeckler and Jokipii (1967), and the more conservative demodulation by Webber (1968). In order to obtain the ionization rate $\zeta$ of $4 \times 10^{-16}$ ionizations per $\mathrm{H}$-atom per sec, mentioned by Field in his report, we need to extend the Gloeckler-Jokipii demodulation down to $10 \mathrm{MeV}$, and we obtain a low-energy cosmic-ray energy density of $1 \mathrm{eV} \mathrm{cm}^{-3}$. For the Webber demodulation these numbers are $2 \mathrm{MeV}$ and $3 \times 10^{-3} \mathrm{eV} \mathrm{cm}^{-3}$. In either case one is multiplying the observed cosmic-ray flux at solar minimum by a very large factor $\left(10^{4}\right.$ and $10^{3}$, respectively).

I would now mention recent work on indirect limits on the flux of low-energy cosmic rays. The results are expressed in terms of $\zeta$. The first limit that $I$ mention is due to Fowler, Reeves, and myself. We have considered the spallation of low-energy cosmic rays in the interstellar medium, producing boron and lithium. The threshold for boron production is $5 \mathrm{MeV}$, and we find that the value of $\zeta$ due to cosmic rays above $5 \mathrm{MeV}$ per nucleon is less than $2 \times 10^{-16} \mathrm{sec}^{-1}$. A similar limit by Solomon and Werner concerns the dissociation of molecular hydrogen by low-energy cosmic rays. The presence of molecular hydrogen in dark clouds, as implied by recent observations by Heiles, indicates that $\zeta$ must be less than, or of the order of, $10^{-16} \mathrm{sec}^{-1}$ for cosmic rays above about $5 \mathrm{MeV}$ per nucleon. A third limit on cosmic rays at still lower energies was obtained by Steigman and myself. We have set limits on the fluxes of heavy nuclei in cosmic rays between roughly 1 and $2 \mathrm{MeV}$. These heavy cosmic rays capture electrons by charge exchange with neutral hydrogen, similar to the cosmic-ray protons, as discussed by Sunyaev. The electrons are captured to excited states, and subsequently cascade to the ground state, emitting resonance line radiation at $\mathrm{X}$-ray energies. Below $1 \mathrm{keV}$ the interstellar medium absorbs the $\mathrm{X}$-rays; and above about $6 \mathrm{keV}$, the abundances of heavy nuclei are too low to produce detectable line 
emission. However, in the 1 to $6 \mathrm{keV}$ range, nuclei such as neon, magnesium, silicon and iron may produce appreciable line emission along lines of sight in the galactic plane. We have applied our predicted fluxes to a recent rocket observation of an excess diffuse X-ray flux in the galactic plane. Unfortunately the measurement has very poor energy resolution, and we find that it is consistent with $\zeta \leqslant 10^{-15} \mathrm{sec}^{-1}$. Improved energy resolution and increased observing time should enable this limit to be considerably lowered in the near future. In the same way, Steigman and I have also recalculated the cosmic-ray proton flux, as discussed by Sunyaev. With more accurate cross-sections we find that a value of $\zeta \leqslant 10^{-12} \mathrm{sec}^{-1}$ would be consistent with the far UV measurements. (Gloeckler, G. and Jokipii, J. R.: 1967, Astrophys. J. Lett. 148, L41; Webber, W.: 1968, Australian J. Phys. 21, 845.)

I should like to consider next the possible role of X-rays in heating the interstellar medium. The diffuse X-ray background extends from $\mathrm{MeV}$ energies to below $1 \mathrm{keV}$. Of importance for the interstellar medium are the soft X-rays. At present there are 5 reported observations of the background at $\approx 0.25 \mathrm{keV}$. Unfortunately, these measurements differ appreciably in the maximum diffuse flux seen, which varies from $\approx 200$ photon $\mathrm{cm}^{-2} \mathrm{sec}^{-1} \mathrm{ster}^{-1} \mathrm{keV}^{-1}$ to $\approx 900$ photon $\mathrm{cm}^{-2} \mathrm{sec}^{-1} \mathrm{ster}^{-1} \mathrm{keV}^{-1}$. For the present purpose, I will simply take the weighted mean of the observations, which yields $\approx 450$ photon $\mathrm{cm}^{-2} \mathrm{sec}^{-1} \mathrm{ster}^{-1} \mathrm{keV}^{-1}$. Werner and $\mathrm{I}$ have considered the effect of this diffuse X-ray flux on the interstellar medium. In particular, we find that the effective ionization rate per hydrogen atom is $2 \times 10^{-17} \mathrm{sec}^{-1}$, about a factor of 20 below the value of $\zeta$ required by Field, Goldsmith, and Habing (see Field's Introductory Report, p. 51). Extrapolation to lower energies of the extragalactic component of the diffuse soft X-ray flux does not increase $\zeta$ significantly.

Higher ionization rates may be obtained using galactic soft $X$-ray sources. A recent experiment by the Wisconsin group (Bunner et al.,1969) sets severe constraints on the number of such sources. Observing one twelfth of the sky, they found no source brighter than 1.3 photon $\mathrm{cm}^{-2} \mathrm{sec}^{-1} \mathrm{keV}^{-1}$ at $\approx 0.25 \mathrm{keV}$. It follows that if soft $\mathrm{X}$-ray sources are to provide a significant heat input to the interstellar medium, there must be either many weak sources or few relatively strong sources. Possible evidence for the first hypothesis is that the same experimenters measured a diffuse flux of $\approx 60$ photon $\mathrm{cm}^{-2} \mathrm{sec}^{-1} \mathrm{ster}^{-1} \mathrm{keV}^{-1}$ at $0.25 \mathrm{keV}$ in the galactic plane. This cannot be due to an extragalactic flux because of the effects of interstellar absorption. In order to obtain a value of $\zeta$ as high as $10^{-15} \mathrm{sec}^{-1}$, it is necessary to postulate that this flux rises steeply towards lower energies. An effective source temperature of about $50 \mathrm{eV}$ is required; interstellar absorption will turn the spectrum over below about $80 \mathrm{eV}$. The net result is a diffuse galactic component with spectrum $\propto E^{2} \exp (-E / 0.05)$ photon $\mathrm{cm}^{-2} \mathrm{sec}^{-1} \mathrm{ster}^{-1} \mathrm{keV}^{-1}$. The number density of sources required is $\approx 10^{-2}$ $\mathrm{pc}^{-3}$, with a luminosity per source of $\approx 10^{32} \mathrm{erg} \mathrm{sec}^{-1}$ in soft X-rays, giving a total heat input to the Galaxy of $3 \times 10^{-26} \mathrm{erg} \mathrm{cm}^{-3} \mathrm{sec}^{-1}$. The corresponding ionization rate $\zeta$, in regions where $n_{e} / n_{\mathrm{H}} \lesssim 10^{-2}$, is $5 \times 10^{-16} \mathrm{sec}^{-1}$ ionizations per $\mathrm{H}$ atom. The distribution of these sources must be that of Population II objects to give the observed degree of isotropy at $0.25 \mathrm{keV}$ when interstellar absorption is taken into 
account. There is indirect evidence that the spectrum of this galactic component must be extremely soft; otherwise, anisotropy would be detectable above $0.5 \mathrm{keV}$, where interstellar absorption is less important. The Wisconsin observers note that no such anisotropy is found as they scan in galactic latitude. For comparison purposes, note that the soft X-ray luminosity of the Sun (averaged over the solar cycle) is $\approx 10^{27} \mathrm{erg} \mathrm{sec}^{-1}$, whereas the soft X-ray luminosity of Sco X-1 (assumed to be at a distance of $100 \mathrm{pc}$ ) is $\approx 10^{35} \mathrm{erg} \mathrm{sec}^{-1}$.

The alternative possibility is to have relatively few strong soft X-ray sources. The main constraint then is that only approximately ten such sources can be brighter than about 1 photon $\mathrm{cm}^{-2} \mathrm{sec}^{-1} \mathrm{keV}^{-1}$ at $0.25 \mathrm{keV}$. This requirement, together with the restriction that these sources not appear abnormally bright above $1 \mathrm{keV}$, leads to the following model. The source luminosity is $\approx 3 \times 10^{37} \mathrm{erg} \mathrm{sec}^{-1}$ in soft $\mathrm{X}$-rays below $0.25 \mathrm{keV}$, and the source density is $\approx 3 \times 10^{-8} \mathrm{pc}^{-3}$. These sources must emit $\approx 3 \times$ $10^{36} \mathrm{erg} \mathrm{sec}^{-1}$ at $0.25 \mathrm{keV}$; therefore, for a thermal bremsstrahlung source, the effective temperature must be $\approx 0.1 \mathrm{keV}$. Observations of soft X-ray sources are still in a very preliminary state. However, the soft X-ray source Vela X-2 may be considered a possible candidate for our hypothetical strong source. Tentative identification of this source with the Wolf-Rayet star $\gamma$ Vel implies a luminosity at $0.25 \mathrm{keV}$ exceeding $3 \times 10^{36} \mathrm{erg} \mathrm{sec}^{-1}$. Moreover, three of the X-ray sources detected at $\approx 1 \mathrm{keV}$ in the Sagittarius region (GX349+2, GX5-1, and GX13+1) show evidence of interstellar absorption consistent with their being at a distance of $\approx 10 \mathrm{kpc}$. Their luminosities are correspondingly estimated at $>10^{36} \mathrm{erg} \mathrm{sec}^{-1}$ above $1 \mathrm{keV}$. The Crab is the only $\mathrm{X}$-ray source for which a reliable distance estimate is available; the luminosity of the $\mathrm{Crab}$ in soft X-rays above $0.01 \mathrm{keV}$ is $\approx 5 \times 10^{37} \mathrm{erg} \mathrm{sec}^{-1}$. (Silk, J. and Werner, M. W.: 1969, Astrophys. J. 158, 185; Bunner, A. N., Coleman, P. C., Kraushaar, W. L., McCammon, D., Palmieri, T. M., Shilepsky, A., and Ulmer, M.: 1969, Nature 223, 1222.)

Sunyaev: Neutral hydrogen in the peripheries of galaxies will be ionized by intergalactic photons at $\lambda<912 \AA$. The ionization will lead to ionized layers around the galaxies, similar to 'Strömgren-spheres' in the Galaxy. $21-\mathrm{cm}$ observations of galaxies can in principle indicate where at the edge of a galaxy a Strömgren-sphere sets in, and from this one obtains an estimate of the intergalactic ionizing flux. Observations of good quality exist for a dozen galaxies. They yield only an upper limit for the intergalactic ionizing flux, but the upper limit is significantly lower than can be obtained from photon counters on board of space probes. The upper limit on the ionizing flux indicates that the density of intergalactic matter is less than one-third the critical density (if the Hubble constant $H=100 \mathrm{~km} \mathrm{sec}^{-1} \mathrm{Mpc}^{-1}$ ). More details may be found elsewhere.* [Sunyaev, R. A.: 1969, Astrophys. Lett. 3, 33; Zel'dovich, Ya. B. and Sunyaev, R. A.: 1969, Zh. eksp. Teor. Fiz. (1970, Soviet Phys. JETP, in translation).]

* See also the criticism by J. E. Felten and J. Bergeron, Astrophys. Lett. 4 (1969), 155. (Ed.) 


\section{The Boundary Layer Between the Stable Gas Phases}

Zel'dovich: Field showed a picture (Figure 3, p. 55), which resembles closely an isotherm in a van der Waals gas. In both cases there are two phases, one with low density and high temperature, the other with high density and low temperature. The two phases have the same pressure. In the case of the van der Waals gas only one pressure is stable, namely that pressure for which the two hatched areas in Figure 1 are equal. This follows from thermodynamic principles. But in interstellar

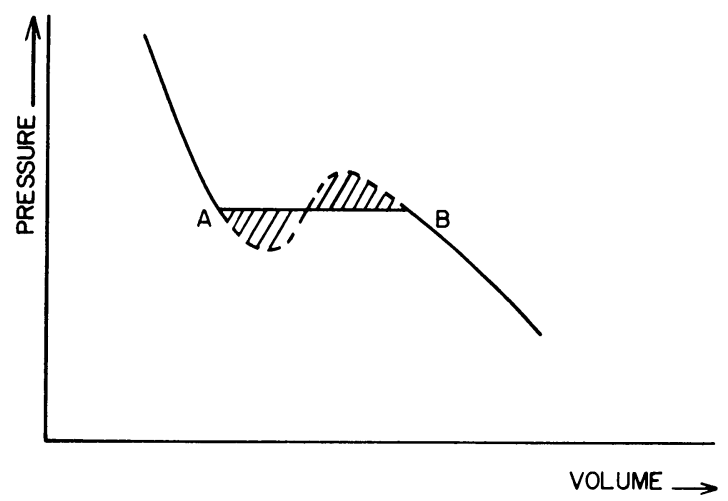

Fig. 1. (See the remark by Zel'dovich.) ( $p-V)$ curve as discussed by Field (p. 55), in which is indicated (schematically) the situation when there is no net heat production in the intermediate layer.

space we do not have closed systems and we cannot apply thermodynamic principles. Still, Pikel'ner and I discovered that also in interstellar space only one pressure is stable. If there is a dense cloud in pressure equilibrium with the rarefied surrounding, then there should be a sheet between them. In this sheet thermal conductivity occurs and has to be considered in addition to cooling and to cosmic-ray heating. The stable equilibrium is determined by the condition that the three processes balance at each point in the sheet. This condition leads to an integral which should be zero; the integral vanishes for only one pressure.* If we have a different pressure, the border sheet will penetrate into the gas in one way or the other. (Zel'dovich, Ya. B. and Pikel'ner, S. B.; 1969, Zh. Eksp. Teor. Fiz.)

Field: Dr. Zel'dovich, I was not aware of the existence of your paper. But just before this Symposium I learned that essentially the same results have been obtained in the United Kingdom by Penston and Brown, apparently independently from you. One thing that emerged from the Penston and Brown paper was that, if the pressure is not the stable one, the conducting boundary layer will move at only an extremely slow rate. In the situation we are considering, Penston estimated a time-scale of $3 \times 10^{8}$ yr, so that in actual situations this equilibrium pressure will not necessarily be found.

* In Figure 1 the cosmic-ray heating exceeds the cooling in the hatched area near $B$; in the similar area near $A$ the reverse is the case. The vanishing of the integral expresses the requirement that the extra heat produced in one part of the sheet is absorbed in the other part. (Ed.) 
Pikel'ner: Indeed, the border moves very slowly through the gas. We concluded that only the initial conditions, and not the physics of the sheet, determine the pressure. For example, suppose that, as in the gravitational theory of spiral structure, gas is condensed into a spiral arm and starts forming clouds (provided that in the interarm region no clouds existed because of the large rarefaction). Then initially the pressure would be the critical pressure (see Field's Figure 3, p. 55 or his Table II, p. 56); but after that the pressure would decrease and the clouds would grow very slowly. Ultimately they would reach the stable pressure, but probably there is not enough time available.

\section{Theoretical Aspects of Interstellar Gas Dynamics and the Formation of Clouds}

Spiegel: Field mentioned a discrepancy between observed and predicted temperatures. Probably this could be explained if the observed gas is not in its final equilibrium. Let me take Figure 3 of Field's paper (page 55) as describing the instantaneous $p-\varrho$ relation. Now consider the idealized case of a uniform layer of height $h$, density $\varrho$ and pressure p. $\varrho$ and $\varrho_{3}$ are the stable equilibrium densities as described by Zel'dovich (see Figure 1 at p. $81 ; \varrho_{1}$ corresponds to point $B, \varrho_{2}$ to point $A$ ), and we have $\varrho_{1}<\varrho<\varrho_{3}$, then the layer is dynamically unstable. If a weight sits on the layer and the system is in a gravitational field, then a slight reduction in $h$ will lead to an initial collapse of the layer. If the loss function adjusts instantaneously, the collapse will maintain the indicated $p-\varrho$ relation. In the phase plane $\varrho-\varrho$, the situation is as shown in Figure 2 a. The point $\left(\varrho=\varrho_{2}, \dot{\varrho}=0\right)$ is a saddle point, while $\left(\varrho=\varrho_{1}, \dot{\varrho}=0\right),\left(\varrho=\varrho_{3}, \dot{\varrho}=0\right)$ are centers. For a perturbation which keeps the loss function constant, the 'orbits' in the phase plane are closed curves, such as the indicated dashed curve. The figure eight curve is the zero energy orbit. Strictly speaking, the motion does not conserve loss rate; and the system will spiral into the appropriate equilibrium. But, if the parameters are correct, we may treat closed oscillating orbits as instantaneously representing the motion. It is to be expected that, in an orbit such as the one indicated, the system spends most of its time near the $\varrho_{2}$ end of the orbit.

Of course, this is an idealization, but this model may indicate the actual behavior much as Baker's one-zone model of stellar pulsation for stars. I would guess that
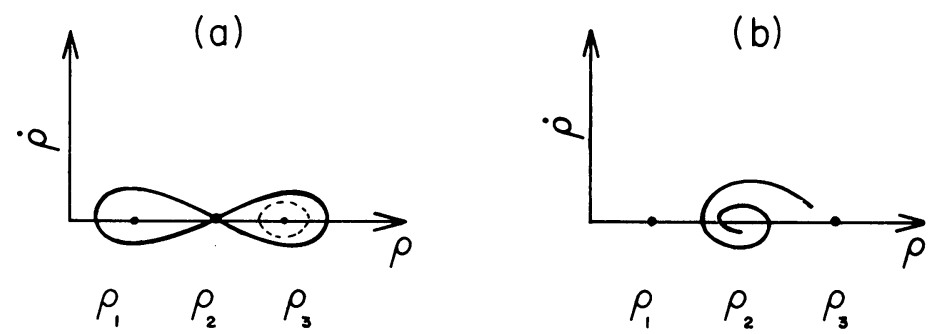

Fig. 2. (See the remark by Spiegel.) Possible orbits in the phase plane of the system considered. (a) Undamped motions. (b) Damped motions. 
it works for individual clouds, and that the majority of clouds are observed not in equilibrium but rather in the kind of damped oscillation indicated by the diagram of Figure $2 b$. Whether we get a limit-cycle or spiraling into a stable point depends on the thermodynamic time scales. The question would have to be studied with timedependent calculations. In this connection, I might mention a thesis by Defouw (1970) at Cal Tech on thermal instability, which shows how thermal instability in a normally stable stratification can lead to convective instability or stability. (Defouw, R. J.: 1970, Astrophys. J. 161, 55.

Field: This is an interesting suggestion. In the solutions by Pikel'ner and Zel'dovich and by Penston and Brown, the boundary layer is so thin that conduction dominates and will stabilize the situation.

Spiegel: Let me raise another subject. The clouds are presumably in the spiral arms. A large number of people believe that those clouds are shocks. What happens when you have a shock in a medium with the equation of state of Figure 1 ?

Field: That problem has been studied by Goldsmith. He made numerical calculations about a piston ramming into the stable hot phase. If one rams it in with more than a certain critical velocity, there is an instantaneous phase transition to the cold stable phase. The critical velocity is such that behind the shock which forms, the pressure exceeds the critical pressure (see my Report, Figure 3, p. 55). The numerical value is about $10 \mathrm{~km} \mathrm{sec}^{-1}$ when the ambient pressure ahead of the shock is not far below the critical value. (Goldsmith, D. W.: 1969, Astrophys. J. 161, 41.)

Pikel'ner: In speaking about the formation of clouds due to thermal instability, we should take into account the magnetic field of the Galaxy. This field is strong, and motions can be only along magnetic lines unless the scale is very large. The time for establishing the equilibrium temperature is short, less than $10^{6} \mathrm{yr}$. After that time we have equilibrium temperatures in different points along the field lines, but not a uniform pressure. Pressure gradients set the gas into motion, and compress fluctuations into clouds. If the diameter of clouds are about $10 \mathrm{pc}$ and motions are possible only in one dimension, then the contraction time may attain $10^{7} \mathrm{yr}$, because rarefied gas is collected out of a very large region. But, if the average density is uniform and we consider the formation of very small fluctuations, then the time will be shorter. However, the observations show rather extensive regions of higher density. Since the time of compression in clouds is long, we can observe regions in which the average density is intermediate between the densities of the rarefied and the dense phase.

Parker: We have to be very careful when we talk about the equation. of state as shown in Figure 1. There is a finite time needed to reach the thermal equilibrium that shows this equation of state, and we have to distinguish between quasi-stationary situations, where the equation of state can be used, and situations with rapid fluctuations in which the thermal equilibrium cannot be reached.

Field: The transition out of the unstable regime is about $10^{6} \mathrm{yr}$. This is one order of magnitude faster than the Rayleigh-Taylor instability which is due to the gravitational field. So, as the latter instability develops, one can get clumping along the magnetic field on a shorter time scale. 
Syrovat-skii: My remark concerns cloud formation. We have heard that the magnetic field is so large that only motion along the magnetic field driven by thermal instability can lead to cloud formation. But if the (strong) magnetic field is not stationary, then a change of the field may produce compression of the gas into a cloud without difficulty. This process may be especially effective near the zero points of the magnetic field, as is shown by examples in the solar atmosphere.

Parker: On a very small scale the magnetic fields inhibit any collapse, just because they are rigid. On a large scale, on the other hand, the magnetic field helps the collapse to proceed. In this context 'large scale' means 'of the order of 100 pc'. 'Small scale' means dimensions of 1 or 2 pc.

Field: It seems to me that Spiegel's suggestion should be studied further, i.e., that the final state of the Rayleigh-Taylor and the thermal instability is in fact a dynamical one. In the Parker model, after the initial instability, the clouds come to rest in the low parts of the magnetic field, unless they are further energized. It is possible that a kind of circulation, such as Spiegel is suggesting, may cause material to evaporate from the dense phase through the unstable phase into the hot phase, and thereby to rise along the field line.Later the material may collapse again to complete the cycle. I raise this point because it seems to me that it is strongly connected with the remark by Weaver (see p. 48) that negative velocities are observed. You see instreaming material; you do not see material going up. Could it be going up in one phase and coming down in another?

Parker: I am intrigued by the suggestion of the continual circulation or dynamical state that Spiegel suggested. There is no ultimate equilibrium that we have been able to discover for the interstellar gas field cosmic-ray system. It seems to me that there is a dynamical balance between the collection of gas into clouds and the disruption of clouds and field by the formation of hot stars and the injection of cosmic rays.

Greenberg: I am trying to connect all this to the discussion on the mass flow into the spiral arms at the Basel Symposium. At the inner edge of the arm, a condensation is produced by a shock wave formed as the mass flows into the potential minimum produced by the density wave. The problem of clouds forming along the spiral arms or along the magnetic fields would then be a separate kind of an instability. In other words, the higher density in the spiral arms is a result of the mass flowing into the potential well, that is the spiral structure, and then the instabilities within the arms produce clouds within the arms. This looks like a consistent picture to me.

Habing: The shock wave that was discussed in Basel is not yet really a physically treated shock. Roberts does not consider what happens perpendicular to the galactic plane. He just considers mass motions in two-dimensional space in the galactic plane.

Pikel'ner: The possibility of formation of a shock wave in the gas flowing into the spiral arms is dependent also on the magnetic pressure. The variation of the transverse velocity of the gas when this gas is flowing into the spiral arms is about 10 to $15 \mathrm{~km}$ $\mathrm{sec}^{-1}$. In the rarefied gas, the magneto-sound velocity should be much higher (about 20 or $30 \mathrm{~km} \mathrm{sec}^{-1}$ if the density is about $0.03 \mathrm{~cm}^{-3}$ and the field $\mathrm{B} \approx 2 \mu \mathrm{G}$ ) and is approximately transverse. Thus the velocity of the gas relative to the spiral arm is 
lower than the sound velocity, and no shock waves should arise. Even if there is no magnetic field, it is necessary to take into account the fact that the gravitational field, which determined the formation of the arms, accelerates the gas gradually.

\section{Observational Aspects of Interstellar Gas Dynamics and the Formation of Clouds}

Ozernoi: In making the choice among mechanisms for obtaining the dense and rarefied regions, it is important to know what kind of correlations are established between the densities, temperatures, velocities, and possibly, the magnetic fields inside and outside the spiral arms, respectively, particularly at high latitudes. I would like Dr. Weaver to comment.

Weaver: That subject was a part of the general review which was deleted in the presentation. I have tried to picture the largest complexes of gas within the Galaxy as spiral arms. Within these arms there are fluctuations in density of various sizes and various temperatures.

To understand in greater detail the kinematical properties of $\mathrm{HI}$ regions of various sizes remains an important observational problem. In studies made so far, inadequate subdivision of cloud sizes has been made. Adequate division is desirable, since there appears to be a correlation between cloud size and other properties of the clouds. I might mention the studies by Takakubo and van Woerden, involving separation of profiles into Gaussian components. Perhaps van Woerden will comment. Again they obtain a picture of an interstellar medium that is not very homogeneous.

Van Woerden: Our study about the kinematics of neutral hydrogen at intermediate galactic latitudes gave the following results. We distinguished two different types of components in the 21-cm profile: (1) Narrow components, with internal velocity dispersions $\sigma$ of the order of $2 \mathrm{~km} \mathrm{sec}^{-1}$, tentatively identified with normal interstellar clouds of densities $n_{\mathrm{H}}$ of the order of $10 \mathrm{~cm}^{-3}$ and of surface densities $N_{\mathrm{H}}$ of $2 \times 10^{20} \mathrm{~cm}^{-2}$ (i.e., sizes of about $5 \mathrm{pc}$ ). Their average distance from the galactic plane was estimated at about 100 pc. (2) Gaussian components with internal velocity dispersions $\sigma$ of the order of 10 to $15 \mathrm{~km} \mathrm{sec}^{-1}$. It appeared that those were at considerably larger distances $(|z|$ about $150 \mathrm{pc})$, and we inferred either that they might form a sort of sheath around the spiral arm, i.e., a transition between arm and interarm region, or that they might possibly occupy a transition region between the disk and the halo. The 'random motions' (external motions of clouds) were about $6 \mathrm{~km} \mathrm{sec}^{-1}$ (r.m.s., radial coordinate only) for both types of component. There was a slight indication for anisotropy in these motions; but the preferential direction varied with galactic latitude, and the relationship of this anisotropy to the local magnetic field was unclear. (Takakubo, K. and van Woerden, H.: 1966, Bull. Astron. Inst. Netherl. 18, 488; Takakubo, K.: 1967, Bull. Astron. Inst. Netherl. 19, 125.)

Verschuur: When van Woerden says he has found narrow components of $2 \mathrm{~km}$ $\mathrm{sec}^{-1}$, it is a reflection of the $2 \mathrm{~km} \mathrm{sec}^{-1}$ bandwidth he used. Correct, Dr. van Woerden? 
Van Woerden: The velocity dispersion of the instrumental profile was $\sigma_{i}=0.8 \mathrm{~km}$ $\mathrm{sec}^{-1}$; so our narrow components were in general not bandwidth-limited. A small number of components were so narrow that they were hard to resolve; but measurements with a narrower band $\left(\sigma_{i}=0.4 \mathrm{~km} \mathrm{sec}^{-1}\right)$ gave essentially the same distribution of dispersions in the interval $0<\sigma<2 \mathrm{~km} \mathrm{sec}^{-1}$, with few if any components having $\sigma<1 \mathrm{~km} \mathrm{sec}^{-1}$. Therefore, our statistics of velocity dispersions were practically not limited by the bandwidth. It may well be that they were limited by instrumental resolution on the sky. If you have a narrower antenna beam, you may get narrower components.

Verschuur: If you use a narrower beam you see components with smaller velocity dispersions, and we really do not know how narrow these things might become as one increases the resolving power. We must be very careful in interpreting data obtained with a small radio telescope, because the interstellar medium has fine-scale structure; and our present picture is biased entirely by the telescopes we have used.

Van de Hulst: I would like to support the last remark. I have the feeling that, at present, analysis is more limited by too low resolutions of the data than by too little thought.

Verschuur: I wish to show data about one distinct cloud in a position-velocity coordinate diagram (see Figure 3). The observations were made with the 300 -foot radio telescope, with a $6.25 \mathrm{kHz}$ bandwidth. Velocity is the horizontal coordinate,
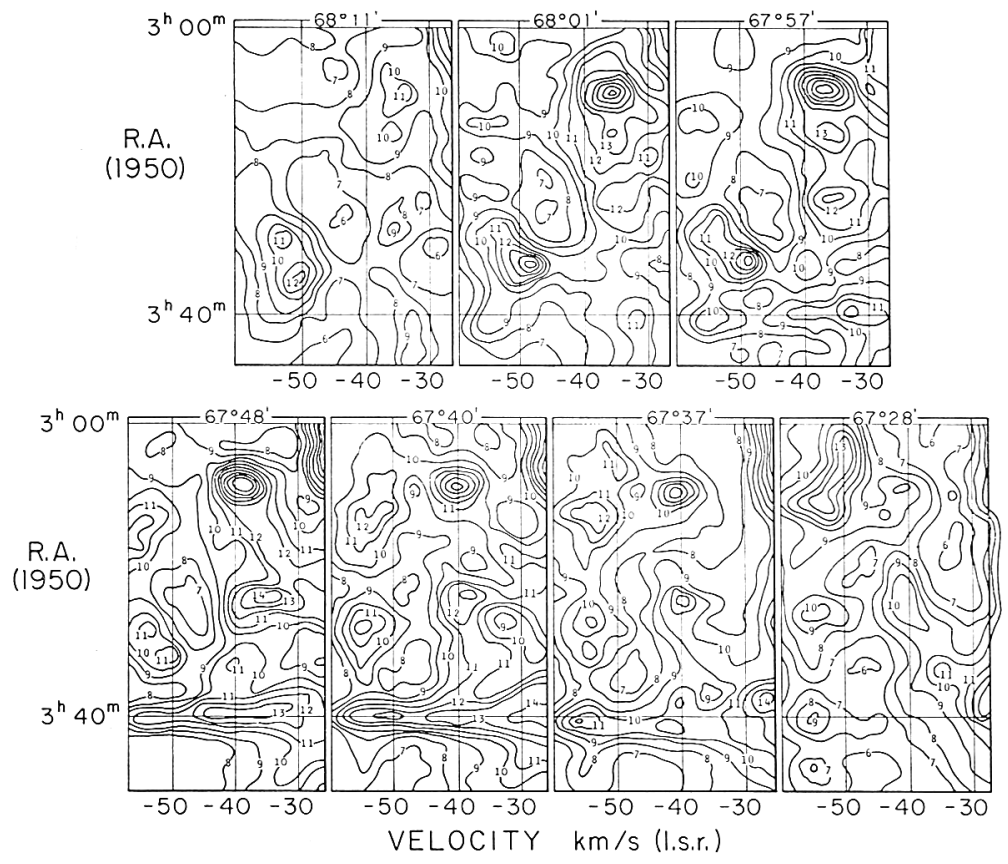

Fig. 3. (See the remark by Verschuur.) 21-cm observations made with the 300-foot radiotelescope at NRAO, Greenbank, W. Virginia (U.S.A.). In each diagram antenna temperatures are plotted in $\mathrm{K}$ as a function of right ascension and velocity; the declination is constant for each separate diagram. 
and right ascension is the vertical. In Figure 3 the declinations are indicated at the top of each section. There is a cloud at about $-40 \mathrm{~km} \mathrm{sec}^{-1}$ and R.A. $3^{\text {h }} 9^{\mathrm{m}}$. It is quite discrete. Its velocity is not associated with either the local or the Perseus spiral arm. The latitude is about $+8^{\circ}$. Notice also that its velocity at declination $68^{\circ} 01^{\prime}$ is $-36 \mathrm{~km} \mathrm{sec}^{-1}$; but half a degree lower in declination, the velocity is $-42 \mathrm{~km} \mathrm{sec}^{-1}$; a change of $6 \mathrm{~km} \mathrm{sec}^{-1}$. I do not know whether it is a galactic or an extra-galactic object. It could be either; but, very clearly, it is a rotating 'cloud'. A discussion of another distinct 'cloud' has been published elsewhere. (Verschuur, G. L.: 1969 Astron. Astrophys. 3, 77.)

Habing: I disagree with Verschuur in regard to the very small features. It is probably true that we shall always find smaller features with larger telescopes. But certainly there are intermediate-scale features in the interstellar gas which are difficult to understand on the basis of a model of discrete, but small clouds. In the $21-\mathrm{cm}$ contours, features exist in which there is continuity over 10 or 15 degrees on the sky.

Burke: Can you give us any numbers?

Habing: In the survey I am making together with Carl Heiles, I find structures extending over some 15 to 30 degrees on the sky. I do not know the distances and cannot therefore give real dimensions. But suppose a feature is 15 degrees long and is some $100 \mathrm{pc}$ away; then the scale length is at least $25 \mathrm{pc}$. You may even have two sheets of ordered motions of gas of $25 \mathrm{pc}$.

Weaver: There is no question whatever about the size of the telescope used influencing the results. But there are plenty of features of large size that present us with many unsolved problems. The larger the telescope, certainly the smaller the feature observed; and we just add more problems to our list. It would be nice to understand even some of the larger clouds.

Menon: What exactly do we mean by 'random motions' of clouds? How can we distinguish between small-scale, local irregular motions, and large-scale, systematic non-circular motion? The data we get cannot be interpreted in the sense of any theories of the general interstellar medium, because we are averaging over regions with very peculiar motions. And we do not know how to account for this averaging.

Weaver: In reply to Menon it appears to me that reasonable approaches exist to the question of the motion of condensations with respect to each other. First the interstellar medium is broken up into rather large-scale units (perhaps described as sections of spiral arms). Within these sections there is a general structure, which observationally appears as condensations in the velocity cross-section of the arm. A first approximation to the velocity dispersion would be to derive the radial velocities of these condensations with respect to the mean of the velocity for the arm. Such an approach is valid, even though the large-scale (arm) structure appears to have some peculiar motion. Such a simple procedure does not take into account differential rotation within the arm, but that could be allowed for.

Thomas: I want to get closer to a definition of clouds. Pikel'ner said that clouds consist of gas in the high-density phase. Weaver essentially talked also about density fluctuations. The picture of two phases goes back to Zanstra at the Second Sympo- 
sium in 1954. We should also remember Adams and Oort and Burgers. Oort and Burgers, in planning the First Symposium asked whether or not the interstellar clouds could be linked to the 'turbulent eddies', in the aerodynamical sense of correlation distances for velocitity fluctuations discussed in the spectral theories. No definite boundaries are necessary to fix scales, only variation in velocity. Although Adams' picture has been conceptually one of discrete entities, it is reasonably clear that he needs only velocity gradients to interpret the observations. One then has to ask whether such gradients can exist in a continuous medium, or whether we require density boundaries. The new aspect the astronomical investigations brought into the discussion is the necessity of incorporating compressible turbulence. One looks for boundaries, not just correlation lengths. This leads to the discussions along Zanstra's lines of quasi-static equations of state, with several phases. I wish that we would try to be clear about what we mean when we say 'clouds'. Are we talking about scales fixed by velocity gradients, or boundaries fixed by density changes, or both? And what is the interrelation? I think Weaver's film shows the beginning of accumulation of data that will answer these questions and will let us define what we mean, operationally, by clouds. Are we talking about smooth fluctuations in a continuum? Or are we talking about discrete entities moving through a very rarefied substratum (with, perhaps, continuum mechanics not providing a good description)?

Habing: From the theoretical side, we are talking about this discrete picture. I disagree with Weaver that the interstellar gas is characterized by a dense gas in which there are gentle fluctuations. My picture is of a rarefied medium filling almost all of the Galaxy. The medium is, however, more concentrated in the arms and, moving into the arms, the gas becomes unstable and forms discrete clouds.

Thomas: Discrete clouds and discrete boundaries, then. Might there be one or more different kinds of clouds?

Habing: There may be clouds of all sizes.

Thomas: Can one therefore have a continuous distribution of clouds, each with different velocity, each with different density, but definite macroscopic velocity for the cloud as a whole?

Habing: Yes. I do not see that this picture is contradicted by the observations, although observationally there is a large-scale ordering, meaning, too, that there are large sheets of clouds.

Weaver: How sharp is the boundary?

Habing: Theoretically, the boundary is determined by conduction, and this would mean a thickness of less than a parsec.

Pikel'ner: We should take into account the magnetic pressures, too. Perpendicular to the magnetic fields, the boundary cannot be really definite; the compression is not very important in this direction. In the direction along the magnetic fields, the boundary should be more definite, perhaps $10^{-3}$ to $10^{-2} \mathrm{pc}$. The other question here concerns the different scales of the condensations. The division into clouds and intercloud medium is dependent on thermal instability. The large scale condensations should be connected with the Rayleigh-Taylor-type instabilities studied by Parker. 
This instability can hardly develop unless it is driven by thermal instability, because the hot gas slides slowly along the magnetic lines. Its height scale is comparable with the height scale of the total layer of the interstellar gas. Thus the thermal instability creates clouds and then these clouds can condense into larger condensations.

Parker: The dynamical theory, involving the magnetic field and the cosmic rays, leads to the prediction that the dimension of clouds in the horizontal direction across the magnetic field may be very small, a few parsecs. It is not clear to me whether or not this effect is observable.

Verschuur: Many clouds are elongated in the direction parallel to the field. I will comment on this in my Report (p. 150).

Mestel: Has anyone considered the effect of galactic differential rotation on the clouds as they form? Other things being equal, the tendency of the differential rotation is to elongate the clouds in the toroidal direction. However, if the arm has a helical magnetic field, the magnetic forces, though not important for the 'grand design' of the spiral structure, may still be strong enough to cancel out effectively the gravitational shear across the spiral arm.

Burke: In this regard I should report one comment by Lin. He maintains that, if the general magnetic field is less than $5 \mu \mathrm{G}$, then the large-scale motion is controlled by gravity and not by the magnetic field.

Karpman: The condensation of clouds is usually accompanied by some dissipative progress, which may unfreeze the matter in the clouds from the magnetic field.

Pikel'ner: The magnetic fields are always important in the interstellar gas. Dissipation can occur only in very sharp neutral lines or shocks, but not during the formation of clouds.

Shulman: I should like to add that not only magnetic fields are an important factor in cloud formation, but also rotation. When the density in a cloud increases, the rotation will be accelerated until it controls the contraction process.

Pikel'ner: Observations of Faraday rotation and some other observations show that magnetic fields are generally parallel. That situation is proof, I think, that rotation is retarded by the magnetic tension.

\section{Observations of the Rarefied, Neutral Intercloud Medium and of the Interstellar Electron Densities}

Van Woerden: Field gave a predicted critical temperature between $7000 \mathrm{~K}$ and $9000 \mathrm{~K}$ and said that the observations yielded a value of $4000 \mathrm{~K}$. Is this difference significant? As far as the observations go, I doubt whether the velocity dispersion, leading to the $4000 \mathrm{~K}$, has been measured accurately since the intensities are low and the background is broad and irregular.

Field: The difference is significant. On the theoretical side it seems impossible to have a stable phase without $\mathrm{L} y-\alpha$ cooling. That requires temperatures of at least $7000 \mathrm{~K}$. On the observational side, the profiles taken by Heiles do not allow more 
than $1 \mathrm{~km} \mathrm{sec}^{-1}$ error in the velocity dispersion. The predicted width is $9 \mathrm{~km} \mathrm{sec}^{-1}$; the observed width, $6 \mathrm{~km} \mathrm{sec}^{-1}$.

Verschuur: I should like to question the temperature of $2000 \mathrm{~K}$ that Weaver obtains for the (neutral) interarm medium. I do not think that one can make a reliable determination from observations with an 85-foot, 35-arc min beamwidth telescope such as he has been using. Observations with a 10 -arc min beam at high latitudes indicate much structure - e.g., many small clouds. At low latitudes the number of small clouds must be much higher, and this makes an interpolation method, as has been used by Weaver, very questionable. I myself have some spectra that show very cold clouds superimposed on a broad background. The broad background has a width consistent with a $7000 \mathrm{~K}$ gas; the cool clouds have kinetic temperatures of $30 \mathrm{~K}$ or less.

Weaver: Certainly there are difficulties in the temperature determination due to an irregular background. You will have noticed that in the diagram I showed (Figure 6, p. 32) the values of the temperature fluctuated considerably. The important point is, however, that nowhere are low temperatures (around $100 \mathrm{~K}$ ) obtained, nor very high temperatures (around $10000 \mathrm{~K}$ ). With respect to the measurement of the broad background that you observed at high latitudes, I think that one has to be very cautious in interpreting the dispersion of the component in terms of temperatures. I frequently found very broad velocity tails, extending to $70 \mathrm{~km} \mathrm{sec}^{-1}$, which have nothing to do with temperature, but only with macroscopic gas motions.

Field: Turbulence can increase the dispersion, but not decrease it. Heiles obtains a dispersion of $6 \mathrm{~km} \mathrm{sec}^{-1}$, so the temperature cannot be more than $4000 \mathrm{~K}$.

Mills: A comment on the distribution of free electrons in the Galaxy. I plotted the positions of 40 pulsars with observed values of the dispersion measure DM on the plane of the Galaxy. I adopted, as a zero-order approximation, a model with a homogeneous distribution of free electrons with $n_{e}=0.06 \mathrm{~cm}^{-3}$. In the plot I recognize two groups of pulsars: one corresponds to the local spiral arm, the other to the Sagittarius arm. The $z$-distribution of the pulsars indicates a mean height of about $100 \mathrm{pc}$ and suggests that the electron layer extends beyond this distance. Details may be found elsewhere. (Mills, B. Y.: 1969, Nature 224, 504).

Weaver: What about possible fluctuations in the electron density?

Mills: Eventually one can detect these by statistical means, but 40 is not a large enough number of pulsars. There seem to be too many electrons in the direction of the Vela X pulsar (perhaps associated with the Gum Nebula), whereas in the direction of the Crab pulsar too few electrons are found.

Field: Recently Davies (1969) discussed the relation between the values of DM and of $N_{\mathrm{H}}$, the total number of hydrogen atoms along the line of sight. Figure 4 shows Davies' data, plotted in a different way. Inside the plot points is written the absolute value of the latitude. If the pulsars were imbedded in a medium with $n_{e} / n_{\mathrm{H}}=0.1$ (an upper limit in the model for the interstellar medium, summarized in my Report), they would all lie on a straight line. However, (i) $N_{\mathrm{H}}$ contains a contribution by hydrogen from behind the pulsar, and (ii) the line of sight may pass through cool clouds 
with considerably lower values of $n_{e} / n_{\mathrm{H}}$. Therefore the observed points are to be expected to lie to the right of the straight line; and, indeed, they are found there. It even seems likely that the lower the galactic latitude, the farther the points are removed from the straight line. Figure 4 then shows that the pulsar dispersion measurements are consistent with the model distribution of interstellar matter mentioned before. (Davies, R. D.: 1969, Nature 223, 355.)

Pottasch: Courtès and Monnet have observed general $\mathrm{H} \alpha$-emission in our Galaxy, confined to within a few hundred pc from the plane. A similar phenomenon was found in other galaxies: a structureless medium on which spiral arms showed up as regions of much higher intensity. Transferring the measurements by Monnet in M51 to our Galaxy (a dangerous thing to do) we have electron densities of $0.1 \mathrm{~cm}^{-3}$ or somewhat less. In addition there is interesting information on the electron temperature. Since, besides $\mathrm{H} \alpha$, the [NII] line is also seen the temperature of the medium is at least $4000 \mathrm{~K}$.

[Editor's remarks: At this point a confused discussion started on various observed quantities (mentioned in the introduction to this Discussion) and their relation to densities and temperatures of the interstellar electrons. The confusion arose mainly

CORRELATION OF DM WITH $\mathrm{N}_{\mathrm{H}}$ FOR 29 PULSARS

(DAVIES, 1969)

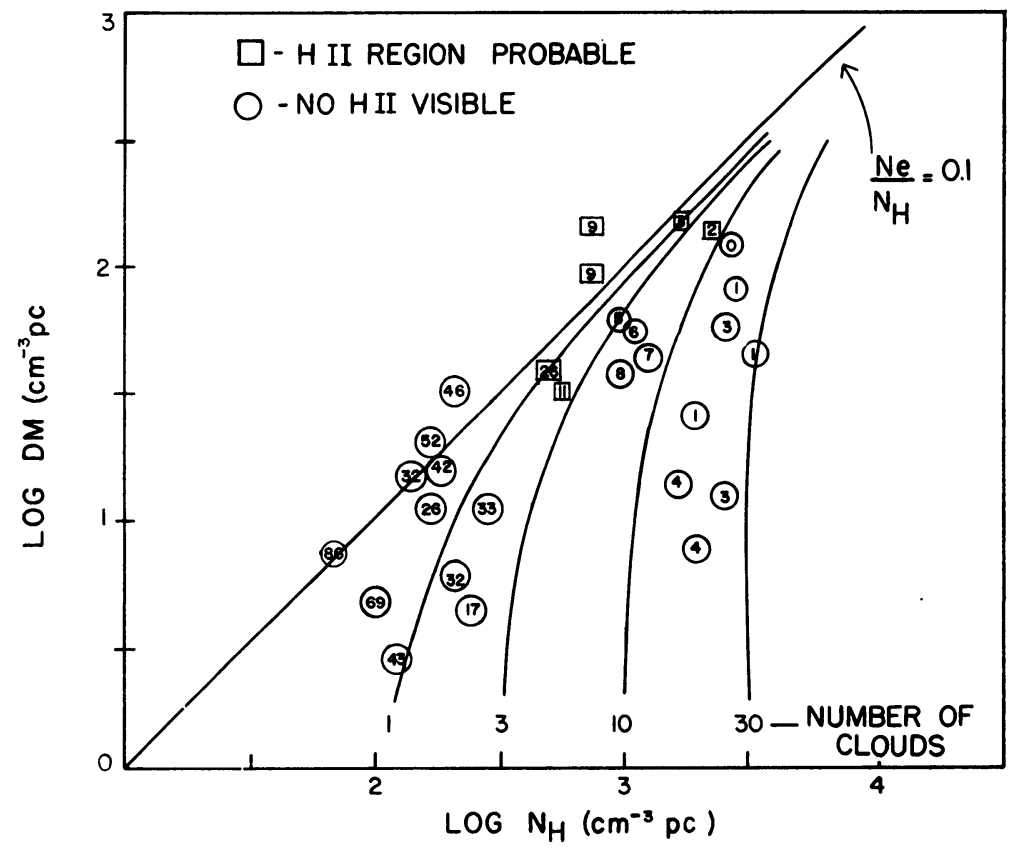

Fig. 4. (See the remark by Field.) Relation between DM, the integral of the electron density along the line of sight, and $N_{\mathrm{H}}$, the analogous integral of the hydrogen density. The data have been taken from Davies, R. D.: 1969, Nature 223, 355. 
because of the complicated, non-linear ways in which most of the observed quantities depend on density and temperature fluctuation. The following conclusions may be extracted from the discussion:

(i) The observed RM- and DM-values indicate that the average interstellar electron density $\left\langle n_{e}\right\rangle$ lies between 0.03 and $0.08 \mathrm{~cm}^{-3}$. This number may be the density in a uniform, rarefied medium; whether this medium is $\mathrm{HI}_{\mathrm{I}}$ or $\mathrm{H}_{\mathrm{II}}$ is an open question.

(ii) 21-cm observations support the existence of a hot, rarefied Hı gas, whose distribution may be somewhat irregular. It seems very attractive to identify this $\mathrm{HI}$-medium with the medium mentioned sub (i), but there is no conclusive evidence for this interpretation.

(iii) Weaver interpreted his $21-\mathrm{cm}$ observations as indicating that in the interarm region the overall $\mathrm{H}$-atom density may be as low as $\frac{1}{40}$ of that inside a spiral arm.

(iv) Mills brought up the paper by Gould (1969), who compared emission and absorption of (radio) bremsstrahlung by galactic interstellar electrons. Gould used mainly data by Wilson (1963) on high-frequency emission and by Ellis and Hamilton (1966) on low-frequency absorption. These data can be explained by a $6000 \mathrm{~K}$ medium with $\left\langle n_{e}^{2}\right\rangle=0.06 \mathrm{~cm}^{-6}$. From the present discussion, two supplemental remarks can be added. First, Gould decided on the temperature of $6000 \mathrm{~K}$ by balancing emission and absorption. If $T$ is higher (say 8000 to $10000 \mathrm{~K}$ ), there is too much absorption compared to the emission, and a model is required consisting of two components with different temperatures. The cool component would provide the absorption; the hot component, the emission. If the cool component is associated with neutral hydrogen clouds, one expects a very irregular distribution of absorption on the sky. This is in contrast to what Ellis and Hamilton state, but it agrees with a remark by Gould about the coincidence of an intense cloud of $\mathrm{HI}$ and a region of unexpectedly large absorption in the survey of Shajn et al. (1961) at $20 \mathrm{MHz}$. The second remark concerns the fact that the observation of bremsstrahlung emission yields (according to Gould) $\left\langle n_{e}^{2}\right\rangle=0.06 \mathrm{~cm}^{-6}$, whereas $\left\langle n_{e}\right\rangle=0.06 \mathrm{~cm}^{-3}$. This implies that the electrons, producing the radio emission, are confined to a small fraction of space (a limiting case is where six percent of space contains $n_{e}=1 \mathrm{~cm}^{-3}$ and the rest of space is empty). Such 'dense' regions (e.g., extended H II regions like the Gum Nebula) also contribute considerably to the low-frequency absorption. In this connection Mezger mentioned that in a high-frequency survey by Altenhoff and Mezger all emission could be attributed to discrete sources (both non-thermal and thermal).

(v) Mills suggested that the $z$-distribution of electrons may resemble more closely the synchrotron disk with $|z|=350 \mathrm{pc}$, than the neutral hydrogen disk with $|z|=$ $200 \mathrm{pc}$. This point has been brought forward by Bridle and Venugopal (1969). It should be mentioned that on basis of the cosmic-ray ionization model such behavior may be expected (at least qualitatively) since the ratio $n_{e} / n_{\mathrm{H}}$ increases with decreasing $n_{\mathrm{H}}$.] (Bridle, A. H. and Venugopal, V. R.: 1969, Nature 224, 545; Ellis, G. R.A. and Hamilton, P. A.: 1966, Astrophys. J. 146, 78; Gould, R. J.: 1969, Australian J. Phys. 22, 189; Shain, C. A., Komesaroff, M. M., and Higgins, C. S.: 1961, Australian J. Phys. 14, 508; Wilson, R. W.: 1963, Astrophys. J. 37, 1038.) 


\section{The Dynamical Theory of HII Regions}

Mezger: I should like to comment on the papers by van de Hulst and Field. It might appear as if the theory and the observations of $\mathrm{HII}$ regions agree and that all problems are solved. This is certainly not the case, and I would be very unhappy if theoreticians would stop working on HiI regions. First, let me mention the problem of electron temperatures. Theory predicts that electron temperatures increase with increasing distance from the exciting star, because of hardening of the ionizing radiation. However, collisional deexcitation counteracts this increase in regions of high density. As a result, in $\mathrm{H}$ II regions with high central densities, we may expect a decrease of temperature in the inner regions, followed by an increase in the outer parts. Observations, however, show only the decrease of temperature. Second, I have observational objections against the theoretical evolution models of $\mathrm{H}$ II regions. These theories start with the following initial conditions: an $\mathrm{O}$ star is turned on in a homogeneous neutral gas. The ionization front advances very rapidly and establishes the initial Strömgren sphere'. Subsequently, the ionization front advances, preceded by a shock front. In later stages, the region is surrounded by a thin, but dense, shell of neutral hydrogen. The theory is well-developed; its only defect is that it considers a situation that has not much relation to reality. Look at some recent observations:

(a) Several independent lines of investigation suggest that the distribution of ionized hydrogen in HII regions is far from smooth. In fact, in young Hil regions, clumping factors (defined as: volume occupied by all ionized gas/total volume of dense regions) are found to be typically of the order of 30. A similar value was found some time ago by Osterbrock and Flather. In recent theories such clumping has been taken into account, to my knowledge, only by Dyson.

(b) In all young $\mathrm{HII}$ regions observed by us at NRAO, the total mass of ionized hydrogen appears to be only a small fraction (less than ten per cent) of the total star mass. Thus, the dynamics and evolution of an $\mathrm{HII}$ region are probably determined by the stars. In fact, it appears as if most of the ionized hydrogen is concentrated around stars.

(c) With only a few exceptions, the ionizing stars of young $\mathrm{H}$ II regions are unknown. Therefore these stars are hidden behind a circumstellar dust cloud. How they can ionize the surrounding gas through the dust cloud is still a puzzle.

So, it appears that the evolution of HII regions is very closely connected with the formation and evolution of star clusters. I will discuss this in more detail in my Report (p. 336).

Field: I think that Mezger is correct in stating that, theoretically, the radiation should become harder as one approaches the ionization front. This hardening will tend to increase the temperature of the ionized gas. But there is a counter-effect that will decrease the temperature, and that has to do with the diffuse Lyman-continuum radiation field. This counter-effect was discovered by Hjellming and has been discussed in my Report. I also agree with Mezger that many $\mathrm{H}_{\mathrm{II}}$ regions are rather irregular, and I admit that density fluctuations pose important theoretical problems. Never- 
theless, it may be going too far to say that all H II regions are irregular. I believe that some appear quite regular. I suggest that these be compared with the theory.

[The following part has been taken from the discussion on Tuesday, September 16 after Thomas requested some reactions from the aerodynamicists present. Dr. Goldworthy's comments were inspired by the question: "How can aerodynamicists contribute to and profit from interstellar gas dynamics?']

Goldsworthy: The dynamic interaction of $\mathrm{H}_{\mathrm{II}}$ regions with density inhomogeneities presents problems to which the aerodynamicist can, I think, contribute greatly. Field suggested in his Report (p. 51) that everything is known theoretically! However, several real difficulties exist on the theoretical side. The most common types of ionization fronts which occur in the expansion of $\mathrm{H}_{\text {II }}$ regions are weak R-type and strong D-type. One need not worry too much about nomenclature; but it should be noted that a weak R-type front corresponds to a weak detonation, and a strong D-type to a strong deflagration in normal combustion theory. These types of combustion fronts do not occur under normal laboratory conditions (the ChapmanJouguet hypothesis). Consequently the expansion of $\mathrm{H}$ II regions presents a phenomenon outside the experience of our terrestrial environment and one in which we are very much interested. Ionization fronts do not occur in the terrestrial environment because here, reaction fronts cannot travel relative to the gas at a rate faster than the thermal sound speed. This limitation does not apply in the interstellar medium where radiative transfer plays a dominant role. Both $\mathrm{D}$ - and $\mathrm{R}$-types of ionization fronts move with supersonic velocity relative to the gas behind them. The principal difficulty facing the aerodynamicist is that for these types of ionization fronts, the two characteristics of the differential equations involved emerge from the back of the ionization front. This situation is in contrast to a shock wave, which travels subsonically relative to the fluid behind, so that one characteristic enters the shock and the other characteristic leaves it. In order to obtain a unique answer to a problem in which weak $\mathrm{R}$ - and strong D-type ionization fronts occur, it is necessary to feed into the calculation the detailed structure of the front. To illustrate the importance of this dictum, I would refer you to the computations carried out by Mathews and referred to by Field in his Report (p. 51). In Mathews' calculations, the shock wave is treated as a diffuse region. In some cases a shock wave occurs within the ionization front. In the numerical treatment this shock wave extends over a major part of the ionization front structure. Clearly, a very drastic approximation is used here and may lead to an incorrect solution to the flow external to the ionization front. If one were to look at the propagation of ionization fronts, considered as discontinuities, one would obtain an infinite number of answers. It is only when you feed in the correct structure that a unique answer is obtained. Thus in undertaking numerical calculations using, say, a total of 100 mesh points, it may be necessary to have perhaps 99 in the structure and 1 elsewhere in the $\mathrm{H}$ II region to obtain consistent results. Essentially what one needs is something akin to the Chapman-Jouguet condition, which is applicable in normal 
combustion theory. That condition has now been obtained for the plane fronts and allows us to solve problems in one space dimension. Inclined ionization fronts constitute a real difficulty, one to which we at the University of Leeds are paying particular attention.

Field: Can you be more detailed in your criticism of Mathews' work?

Goldsworthy: The real difficulty is that, while Mathews does feed structure calculations into his numerical procedure, and everything is all right in the early stages, the results go wrong when the front reaches about twice the thermal sound speed of the Hil region. This is precisely the point at which D-type ionization fronts can occur. The problem is aggravated for the higher-powered stars. As I have already mentioned, one must be very sure that the correct structure is being fed. What happens is that at some stage a shock is formed within the ionization front. In Mathews' calculations, he obtains a velocity of a weak R-type front of about the same magnitude as the sound speed; more refined structure calculations show that this is not possible. What is more, if one wants to go further with the calculations, one is in trouble because the range of influence extends from the place where the calculations went wrong. The errors were in the final stages of Mathews' calculations; if he had gone further, he would have obtained completely wrong and meaningless answers.

Field: Would the detached shock have a velocity higher or lower than the velocity that Mathews calculated?

Goldsworthy: I would guess that the shock which goes in front would have a lower velocity, because precisely at the place where the weak R-type front occurs in Mathews' calculations you are more likely to have a strong D-type which is slower moving. The difference would be small, in the Hi region, but large in the flow in the Hil region.

Field: My second question concerns the discrepancy between the calculations of the shock, and what seems to have been observed by the radio astronomers. The calculations indicate that the thickness of the HI region between the ionization front and the shock front is between 1 and 10 per cent of the radius of the H II region, depending upon the assumed thermal cooling time. On the other hand, the radio observations consistently give 50 per cent for this parameter in six cases. I wonder whether or not you would consider this a serious discrepancy from the theoretical viewpoint, in the light of the uncertainty you have just stated? Later we should ask the observers whether or not these data are reliable.

Goldsworthy: My comments would not make much difference with respect to the distance between the shock in the $\mathrm{HI}$ region and the ionization front. No matter what one does aerodynamically, it is difficult to let the shock move away from the ionization front. It is therefore a problem of interpretation of the data; otherwise, we have to think of some other process that will separate shock and ionization front.

Van Woerden: Dr. Field, can you elaborate on the six cases you mentioned?

Field: The technique has been to scan across a number of Hil regions in the $21-\mathrm{cm}$ line, using a telescope with a beam of about $1^{\circ}$. The expansion velocity of the $\mathrm{HI}$ region is deduced from the splitting of the $21-\mathrm{cm}$ line, along the line of sight through the center of the Hil region. The angular extent of the expanding Hi shell is judged by 
the variation of the $21-\mathrm{cm}$ profile across the Hil region. This judgment, however, is difficult and problematic according to Riegel (1967). I do not think we can solve the observational difficulties here. I am only asking the observers to re-examine the problem with a view to testing the presented theories. (Riegel, K. W.: 1967, Astrophys. J. 148, 87).

[The following part has been taken from the discussion on Thursday, September 18.]

Toomre: Dr. Mezger, you seem to reject any observational evidence for expanding $\mathrm{HI}$ shells surrounding $\mathrm{H}$ II regions. Could you give more detail?

Mezger: By correlating the space-velocity distribution of neutral ( $\mathrm{HI})$ and ionized (HII) hydrogen one sees immediately that $\mathrm{HII}$ regions are located where the $\mathrm{HI}$ gas shows a maximum density. In other words, massive stars form in the centers of dense clouds of $\mathrm{HI}$ gas. Consequently one would expect to find $\mathrm{HI}$ regions surrounded by $\mathrm{HI}$ gas of relatively high density and that is what single-dish observations of some Hil regions show. However, these are not the relatively thin shells of highly compressed neutral gas which have been predicted to precede an advancing ionization front. Either uniformly expanding $\mathrm{H}$ II regions surrounded by dense shells of neutral gas do not exist or the angular resolution of present $21-\mathrm{cm}$ telescopes $\left(\gtrsim 10^{\prime}\right)$ is not sufficient to detect them. We shall probably have to wait for interferometer $21-\mathrm{cm}$ line observations to give this answer.

Van Woerden: To name but one example, in the Lacerta association, Sancisi and I (unpublished) have found clear evidence for neutral hydrogen moving away from the stars towards us with a velocity of about $10 \mathrm{~km} \mathrm{sec}^{-1}$.

Mezger: Would you interpret it as an expanding shell, or is it just one cloud which is moving away from the star?

Van Woerden: It is not a complete shell around the association. I think it is much too idealized a picture to expect a complete spherical shell. But we do see an extended cloud, or whatever you want to call it, with a velocity of $10 \mathrm{~km} \mathrm{sec}^{-1}$ coming at us.

Mezger: Is there an Hil region observed in this association, e.g., as a thermal radio source?

Van Woerden: I do not know of radio observations, but optical observations have shown an Hil region.

Field: Perhaps we cannot settle now this question of the observations of the $\mathrm{HI}_{\mathrm{I}}$ shell. But I am interested in hearing Mezger say that it really is a question of having sufficient resolution. So, there is no evidence against such expansion; rather we might say that there simply is no evidence at all on the question one way or the other.

[The following remark was made at the Tuesday, September 16 discussions.]

Goldsworthy: There is another point $\mathbf{I}$ wish to draw attention to. We know that inhomogeneities exist in interstellar gas clouds. We can learn much about the dynamics of ionization fronts by their interaction with such inhomogeneities. I remind you of 
the work of Dyson (1968) on globules. Suppose one takes a high density globule illuminated by some radiating star (the situation which Mezger accused theoreticians of evading). With low-density gas outside, we imagine that their fronts will move quite rapidly. Probably a fast moving weak R-type front will result. This front will meet high density material. What sort of flow is established? If the density of the 'globule' is not too much higher than the gas outside, the gas inside will be heated to the same temperature as that outside; but the higher density of gas in the globule means a higher pressure. A motion is therefore established which is akin to that in a shock tube. In other words, shocks will be sent out from the object, and rarefaction waves will traverse the high density gas within. This rarefaction wave will weaken the expanding shocks most at the place where the ionization front interacts first with the 'globule'. This weakening of the shock governs the length of time the object is seen in emission within Hil regions. The important point to note is that, associated with these objects, there will be sideways shocks carrying the material out with it. If the globule is of high density, the ionization front will be curved, thus running into the difficulties already mentioned of having more than one space dimension involved. Approximative theory is available, however; and in this case the 'hypersonic' approximation is a very useful tool for discussing these problems. (Dyson, J. E.: 1968, Astrophys. Space Sci. 1, 388). 